\title{
Effect of Soil Mulching and Crop Residues on Enhancement of Cucumber Resistance to Some Soil Borne Diseases Abeer M.A. El-Hadidy
}

Plant Pathol. Unit, Plant Protection Dept., Desert Res. Centre.

\begin{abstract}
The application of crop residues as soil amendments followed by soil plastic mulching is considered an environmentally friendly approach for the control of soil borne plant pathogens. The objective was to evaluate the effectiveness of this approach in Egypt. To this aim, a plastic greenhouse experiment was carried out to compare the effects of plastic mulching, alone/or in combination with five different crop residues on root-rot and wilt of cucumber caused by Pythium ultimum and Fusarium oxysporum f.sp. radices-cucumerinum, respectively. Crop residues, i.e. alfalfa, mustard, canola, cabbage, cauliflower and radish were incorporated into natural infested soil and covered with plastic film for 60 days. After this time, cucumber plants were planted and inoculum survival evaluated. During crop development, disease incidence and severity were assessed; the application of crop residues followed by plastic mulching did manage to negatively affect Pythium sp. and Fusarium spp. inoculum survival. Nonetheless root-rot and wilt disease incidence were significantly reduced by $80-90 \%$ of cabbage, radish and cauliflower residues followed by plastic mulching. The application of crop residues increased plant growth and yield compared to untreated controls. These results suggest that, crucifer (Brassica sp.) residues followed by plastic mulching reduced the incidence and severity of cucumber rootrot and wilt diseases under plastic greenhouse conditions
\end{abstract}

Keywords: Biofumigation, crop residues, cucumber, Fusarium wilt, Pythium, root-rot and solarization.

Cucumber (Cucumis sativus L.) is one of the most important crops in Egypt. Under plastic-houses conditions. Root-rot and wilt diseases are commonly encountered in the plastic-houses and are primarily caused by the ubiquitous pathogens, Pythium ultimum and Fusarium oxysporum.

Soil disinfestation treatments, primarily utilizing biocidal chemicals or various forms of heat, are used to reduce soil borne inoculum of crop pests including fungal, bacterial, and nematode pathogens.

These effects afford protection and stimulation of root growth and crop yield and are often interrelated through complex mechanisms involving drastic qualitative and quantitative changes in the soil environment (Chen et al., 1991).

Soil solarization is an approach of soil disinfestation, where passive solar heating for moist soil mulched with plastic sheeting (transparent polyethylene) by exposure to sunlight during the hottest months were used. This process changes soil physical, chemical and biological properties and thereby helps to improve soil health 
(Stapleton, 2000). Soil solarization has been widely utilized in arid areas, such as Egypt, India, Iran, Jordan and Syria. Under conductive conditions and proper use, solarization can provide excellent control of soil borne pathogens in the field, greenhouse and nursery.

Solarization's mode of action is complex, involving direct thermal destruction of propagules, shifts in microbial populations and activity, in addition to changes in the soil's physical and chemical properties. Because solarization is a passive, meteorologically dependent process, integration of other physical, chemical, and biological control methods is desirable for improvement of efficacy and predictability of pathogen control (Stapleton et al., 1991). Under marginal environmental conditions with thermotolerant pest organisms or those distributed deeply in soil, or to minimize treatment duration, it is often desirable to combine solarization with other appropriate pest management techniques in an integrated pest management approaches to improve the overall efficacy of treatment (Stapleton, 1997). Solarization can be combined with a wide range of organic amendments, such as composts, crop residues, green manures and animal manures to increase the pesticidal effects of the combined treatments (Gamliel and Stapleton 1993a; Chellemi et al., 1997 and Klein et al., 2011). Incorporation of organic amendments, including dried, ground crop residues is nonchemical approach to improving the efficacy and predictability of some soil borne pathogens control by solarization. They have attributed the improved control to enhanced production of volatile from the amendments (Gamliel and Katan, 2009 and Klein et al.; 2007 and Klein et al., 2011). Combining these materials with solarization can greatly increase the biocidal activity of the amendments. The concentration of many volatile compounds emanating from decomposing organic materials into the soil atmosphere have been shown to be significantly higher when solarized (Gamliel and Stapleton 1993b; Stapleton, 2000 and Berbegal et al., 2008).

Biofumigation is an agronomic practice of using volatile chemicals (allelochemicals), released from decomposing crop residues, to suppress soil borne pathogens (Matthiessen and Kirkegaard, 2006; Berbegal et al., 2008; Anita, 2012 and Hansen and Keinath, 2013). It also has been used as an alternative to methyl bromide and other synthetic fungicides in agriculture in general. It has also been used to reclaim soils contaminated with heavy metals (Karavina and Mondumbu, 2012). Brassica residues are mainly used for the potential of secondary plant products released from residues of Brassica spp. Decomposition of plant tissues in these families releases isothiocyantes which are biocidal.

The biocidal activity of various isothiocyantes released by Brassica tissues is well-known for its potential to suppress a range of soil borne pests and diseases (Anita, 2012).

Incorporation of these organic materials by themselves may act to reduce numbers of soil borne pests in soil by altering the composition of the resident microbes or of the soil physical environment (Berbegal et al., 2008).

Egypt. J. Phytopathol., Vol. 41, No.1 (2013) 
This study aimed to compare the effects of crop residues alone and in combination with solarization on cucumber root-rot and wilt diseases under commercial plastic greenhouses.

\section{Materials and Methods}

Isolation, Identification and pathogenicity test of the causal fungi:

Samples of cucumber plants showing root-rot and wilt symptoms collected from different commercial plastic greenhouse locations, i.e. Noubariya, El-Bostan, El-Khatatba and Salhiya districts, were subjected to isolation trials. Specimens of diseased cucumber roots were aseptically dissected, placed on PDA plates and incubated at $28^{\circ} \mathrm{C}$ for up to 5 days. The emerged fungi was picked up on PDA plates and purified. Pure cultures were identified according to the description of Gilman (1957) and Barnett and Hunter (1998).The purified isolates, i.e. Pythium ultimum, Rhizoctonia solani, and Fusarium oxysporum, were tested for their pathogenicity on cucumber under greenhouse conditions. Plastic pots $(25 \mathrm{~cm})$ containing sandy loam soil were artificially infested with the inoculum of any tested fungi at the rate of 5\% by weight. The inoculum was prepared by sowing each tested fungus on sand: barley medium $(1: 1, w / w$ and $40 \%$ water $)$ for two weeks at $25^{\circ} \mathrm{C}$. After words the cucumber seeds were sown at the rate of $6 \mathrm{seed} /$ pot. Six pots were used as replicates for each treatment as well as cheek treatment (non-infested soil). Disease development was observed during the growth period for up to 60 days. The percentage of pre and post emergence damping-off were assessed 15 and 40 days after sowing, respectively.

\section{Crop residues preparation:}

Leaves and stem debris of the following plants were tested: mustard (Brassica juncea L.), canola (B. nupus L.), cabbage (B. oleracea L. var. capitata), cauliflower (B. oleraceae L. var. compacta), radish (Raphanus sativus L.) and alfalfa (Midicago sativa). The foliages of these crops were collected from commercial agricultural fields during crop production. The leaves and stems of each crop were separately air dried at $25-30^{\circ} \mathrm{C}$ for 10 days, then ground and sieved through a $2 \mathrm{~mm}$ sieve. The sieved residues of each plant species were used as soil amendments.

Field experiment:

Field experiment was conducted during summer season in 2009 in commercial plastic greenhouse at Noubariya district, to evaluate the effect of crop residues, solarization and/or combination with its. Sandy loam soil was used (sand 91.2\%, silt $3.70 \%$, clay $5.10 \%$, organic matter $0.30 \%$, N 5.10 ppm, P 3.20 ppm and K 20 ppm) which was naturally infested with $P$. ultimum, $R$. solani and $F$. oxysporum f.sp. radices-cucumerinum.

Soil was divided into stripes having $120 \mathrm{~m}^{2}$ areas per each treatments were arranged in split plot design with three replications. Dried and ground crop residues amendments, were incorporated into soil at a rate of $2 \%(\mathrm{w} / \mathrm{w})$ as indicated (equivalent to a rate of $2 \mathrm{~kg} / \mathrm{m}^{2}$ in the field and mixed to a depth of about $15 \mathrm{~cm}$ in the soil with a Rot tiller. Soil was irrigated to a depth of $80 \mathrm{~cm} 3$ days before application of the translucent plastic covers. 
The strips treated with solar heating were mulched with a transparent polyethylene sheet (150 m thick) very closed to the soil surface with keeping their edges anchored in trenches along the strip sides to start the solarization treatments, with keeping some stripes without covering as control. Uncovered stripes were irrigated at 3 days intervals to retain soil moisture. The following treatments were exposed to full sunlight: soil mulching plus crop amendments; crop amendments (no soil mulching); no crop amendments (soil mulching) and both no crop amendments no mulching (untreated control). The solarized stripes were exposed to solarization for a period of 60days during the months of July and August 2009, as indicated. Soil temperatures were recorded at several depths $(5-10-20-\mathrm{cm})$, it was measured using a digital electronic stem thermometer.

Cucumber transplants (Cucumis sativus L.) were sown on $1^{\text {st }}$ October with no-till after finishing the solarization treatments at 10 seedlings within each row. Cucumber seeds were sown 15 days before transplanting in tube preformed trays containing peat/vermiculite (1:1, v:v). Incidences of rootrot disease were recorded after 4 weeks from transplantation.

Plant samples were taken at 45 days to determine root-rot and wilt diseases, also some growth characters of cucumber, i.e. plant height, fresh weight, dry weight and yield, were determined.

Diseases assessment:

The percentages of infected plants with wilt and/or root-rot were recorded up to 8 weeks after transplanting as follows:

$$
\text { Infection }(\%)=(\text { No. of infected plants / plants }) \times 100 \text {. }
$$

Wilt development on each plant was rated using the scale described by Gao et al. (1995) as follows: 5=plant dead; 4= 76 to $100 \%$ of leaves with symptoms, $3=51$ to $75 \%$ of leaves with symptoms, $2=25$ to $50 \%$ of leaves with symptoms; $1=$ less than $25 \%$ of leaves with symptoms and $0=$ no symptoms. The disease rating was calculated by the following formula:

$$
\text { Disease index }=\frac{\sum \text { (Rating No. } x \text { No. of plants in the rating) }}{\text { Total No. of plants } \mathrm{x} \text { highest rating }}
$$

Internal symptoms were determined based on length of vascular discoloration (cm) as described by Szczech (1999).

\section{Soil biological assays:}

Soil samples were collected from the upper layer (20-cm depth) of solarized and non-solarized soil at four dates: 1) immediately, pretreatments (0 time); 2) after plastic removal (60 days after treatments); 3) two months after cucumber were planted (150 days after treatments); 4) at the end of the growing season (180 days after treatments).

Population densities of selected soil microorganisms and inoculum density of pathogens were estimated by dilution plating to monitor the effects of soil solarization with crop residues. Four $2.5 \mathrm{~cm}$ diameter cores collected from $15 \mathrm{~cm}$ depth from the centre row of each subplot were composited and thoroughly mixed. 
A suspension of $10 \mathrm{~g}$ of soil in $90 \mathrm{ml}$ of sterile deionized water was serially diluted and $0.1 \mathrm{ml}$ of soil dilutions was plated into three plates of different selective media, then Komada's medium (Komada, 1975) was used to isolate Fusarium spp.; Gallic acid medium (Flowers and Hendrix 1969) was used to isolate Pythium sp; Potato dextrose agar medium (PDA) containing $50 \mathrm{mg}$ of chlortetracycline hydrochloride per litre of medium (Marois et al., 1981) was used to isolate fungi; modified Bunt and Rovira medium (Abd El-Hafez, 1966) was used to isolate bacteria and Starchnitrate agar medium (Waksman, 1957) was used to isolate actenomycetes. Plates were incubated at $28^{\circ} \mathrm{C}$ for $2-4$ days, when the developed individual colonies were examined and counted.

Statistical analysis:

Data were subjected to analysis of variance (ANOVA) and were calculated for mean separation analyzed and subjected to Duncan's multiple range test and comparison after analysis of variance (Duncan, 1955).

\section{R e s u l t s}

Survey, isolation, and identification of the causal fungi:

A survey study was carried out to detect the main fungi associated with root-rot and wilt symptoms of cucumber seedlings and plants in five sits of commercial plastic houses, i.e. Noubariya, Salhiya, El-Bostan, El-Khatatba and Banger El-Soker. Isolation of the pathogenic fungi was performed from roots at different stages of plant growth (Table1). Data showed that, Pythium spp., Fusarium solani, Rhizoctonia solani, Fusarium oxysporum and Sclerotium rolfsii were the most prevalent fungi isolated from infected cucumber roots. Data revealed that, the highest infection with root-rot and wilt was found in Noubariya (54.3\%) and Salhiya (46.2\%), respectively, while the lowest infection was found in El-Khatatba (27.5\%). Isolation fungi from diseased plants indicated that Pythium ultimum was the most dominant fungi, it was also observed that $P$. ultimum and $F$. oxysporum were highly presented in plants growing at Noubariya (34.2\%) and $(32.3 \%)$, respectively, followed by other sites. Nevertheless, results indicated that $R$. solani was highly presented at Salhiya $(26.4 \%)$ followed by other sites.

Table 1. Frequency of isolated fungi from root-rot and wilt cucumber plants cultivated under commercial plastic houses

\begin{tabular}{|l|c|r|r|r|r|r|r|}
\hline \multirow{2}{*}{ Location } & Infection & \multicolumn{6}{|c|}{ Isolated fungi frequency (\%) } \\
\cline { 4 - 9 } & $(\%)$ & $\begin{array}{c}\text { Rhizoctonia } \\
\text { solani }\end{array}$ & $\begin{array}{c}\text { Fusarium } \\
\text { solani }\end{array}$ & $\begin{array}{c}\text { Pythium } \\
\text { spp. }\end{array}$ & $\begin{array}{c}\text { Sclerotium } \\
\text { rolfsii }\end{array}$ & $\begin{array}{c}\text { Fusarium } \\
\text { oxysporum }\end{array}$ & Others \\
\hline Noubariya & $54.3 \mathrm{a} *$ & $16.6 \mathrm{~b}$ & $13.4 \mathrm{a}$ & $34.2 \mathrm{a}$ & $2.7 \mathrm{a}$ & $32.3 \mathrm{a}$ & $5.8 \mathrm{~d}$ \\
\hline Salhiya & $46.2 \mathrm{~b}$ & $26.4 \mathrm{a}$ & $12.2 \mathrm{a}$ & $25.3 \mathrm{~b}$ & $1.2 \mathrm{~b}$ & $17.5 \mathrm{~b}$ & $9.4 \mathrm{~d}$ \\
\hline El-Bostan & $37.6 \mathrm{c}$ & $16.5 \mathrm{~b}$ & $9.7 \mathrm{a}$ & $20.6 \mathrm{~b}$ & $2.5 \mathrm{a}$ & $13.2 \mathrm{~b}$ & $37.5 \mathrm{~b}$ \\
\hline El-Khatatba & $27.5 \mathrm{~d}$ & $13.6 \mathrm{~b}$ & $11.5 \mathrm{a}$ & $12.5 \mathrm{c}$ & $1.5 \mathrm{~b}$ & $11.3 \mathrm{~b}$ & $49.6 \mathrm{a}$ \\
\hline Banger El-Soker & $43.5 \mathrm{~b}$ & $17.4 \mathrm{~b}$ & $10.9 \mathrm{a}$ & $26.4 \mathrm{~b}$ & $1.7 \mathrm{~b}$ & $13.4 \mathrm{~b}$ & $27.2 \mathrm{c}$ \\
\hline
\end{tabular}

* Values followed by the same letter in each column are not significantly different at $\mathrm{P} \leq 0.05$ according to Duncan's multiple range tests. 
Pathogenicity test:

Results presented in Table (2) indicate that $R$. solani, $P$. ultimum and $F$. solani caused the heights root-rot of cucumber plants, four weeks after transplanting, while after eight weeks, the infection percentage of root-rot caused by $R$. solani, $F$. oxysporum, $P$. ultimum and $F$. solani were clearly high by $67,9,66.5,64.7$ and $63.6 \%$, respectively. These isolates were identified as P. ultimum (Edson) Fizp., $F$. oxysporum f.sp. radices-cucumerinum Schlecht, $S$. rolfsii Sacc., $R$. solani Kuhn and F. solani (Mart.).

Table 2. Percentage of root rotted and wilted cucumber after transplanting in soil artificially infested with different soil borne fungi

\begin{tabular}{|l|c|c|c|}
\hline \multirow{2}{*}{ Tested fungus* } & \multicolumn{2}{|c|}{ Infection $(\%)$} & $\begin{array}{c}\text { Survived plants } \\
(\%)\end{array}$ \\
\cline { 2 - 3 } & 4 weeks & 8 weeks & $30.4 \mathrm{~b}$ \\
\hline Rhizoctonia solani & $58.3 \mathrm{a} * * *$ & $67.9 \mathrm{a}$ & $34.3 \mathrm{~b}$ \\
\hline Pythium ultimum & $51.6 \mathrm{a}$ & $64.7 \mathrm{a}$ & $34.9 \mathrm{~b}$ \\
\hline Fusarium oxysporum & $24.2 \mathrm{c}$ & $66.5 \mathrm{a}$ & $39.6 \mathrm{~b}$ \\
\hline Fusarium solani & $44.3 \mathrm{~b}$ & $63.6 \mathrm{a}$ & $50.8 \mathrm{~b}$ \\
\hline Sclerotium rolfsii & $31.4 \mathrm{c}$ & $34.9 \mathrm{~b}$ & $100 \mathrm{a}$ \\
\hline Non-infested & $0.0 \mathrm{~d}$ & $0.0 \mathrm{~b}$ & \\
\hline
\end{tabular}

* Soil was infested by each fungus at rate of $5 \mathrm{~g} / \mathrm{kg}$ soil before seedling transplanted.

** Data were recorded 4 and 8 weeks after transplanting.

*** Values followed by the same letter in each column are not significantly different at $\mathrm{P} \leq 0.05$ according to Duncan's multiple range tests.

Effect of solarization treatments on soil temperature:

Generally, soil temperatures were exceeded as a result of soil polyethylene sheet mulching during the solarization period ( $1^{\text {st }}$ July to $30^{\text {th }}$ August) of summer season 2009, as illustrated in Fig. (1). Maximum soil temperatures were higher in the solarized plots if compared with the non-solarized ones. The maximum soil temperature at $5 \mathrm{~cm}$ depth reached $51^{\circ} \mathrm{C}$ under sheet alone, while, it was $46.6^{\circ} \mathrm{C}$ at $10 \mathrm{~cm}$ depth and $43^{\circ} \mathrm{C}$ at $20 \mathrm{~cm}$ depth. Meanwhile, it didn't exceeded $38^{\circ} \mathrm{C}$ in non-solarized plots. Likewise, the maximum soil temperature at $5 \mathrm{~cm}$ depth reached $55^{\circ} \mathrm{C}$ under sheet with residues, while, it was $51^{\circ} \mathrm{C}$ at $10 \mathrm{~cm}$ depth and $47^{\circ} \mathrm{C}$ at $20 \mathrm{~cm}$ depth. Soil temperatures showed significant increase as a result of elongating incubation period.

Effect of solarization or/and crop residues on cucumber root-rot and wilt incidence:

Results in Table (3) and Fig. (2) indicate that incorporation of all crop residues significantly reduced the percentage of infection and disease severity for both rootrot and wilt diseases. The most significant effective treatments were cabbage and radish to reduce infection percentage of root-rot by 66 and $58.3 \%$, respectively, in non-solarized soil compared with untreated control. However, incorporating radish, cauliflower and cabbage as crop residues in non-solarized soil led to suppress incidence of Fusarium wilt by $75,70.1$ and $69.3 \%$, respectively, compared with untreated control. 


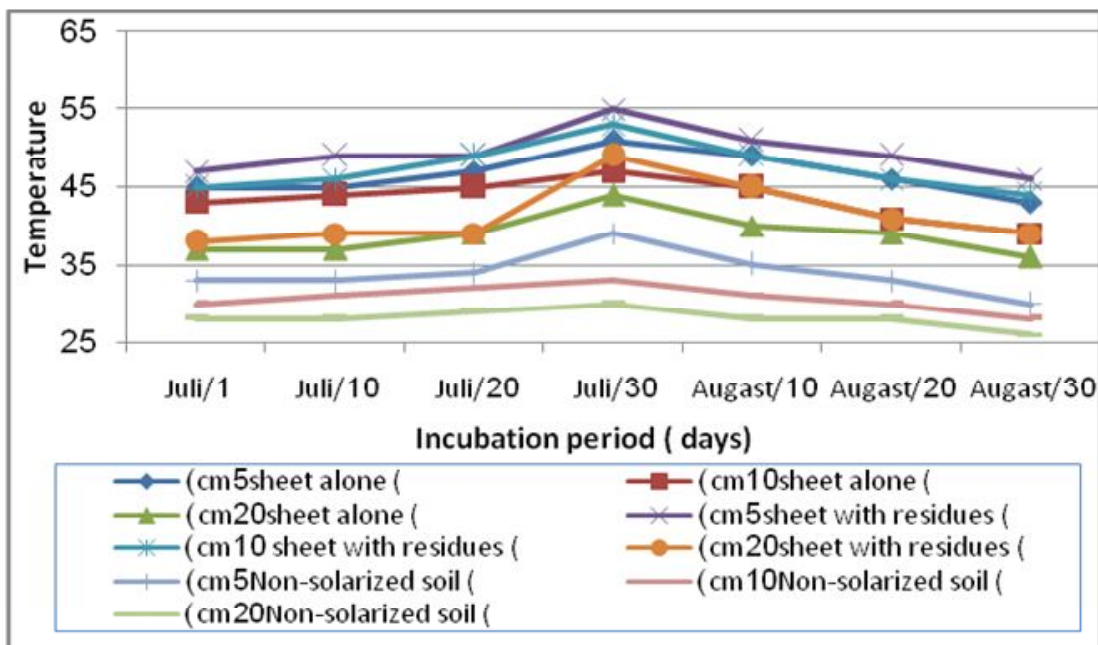

Fig. 1. Daily temperature at three depths in solarized and non-solarized treatments during the hot summer days.

Table 3. Effect of combination of solarization and crop residues treatments on incidence of root-rot and wilt of cucumber plants grown under plastic house

\begin{tabular}{|c|c|c|c|c|c|c|}
\hline \multirow[b]{2}{*}{ Treatment } & \multirow[b]{2}{*}{$\begin{array}{c}\text { Crop } \\
\text { residues }\end{array}$} & \multicolumn{2}{|c|}{ Pythium root-rot } & \multicolumn{3}{|c|}{ Fusarium wilt } \\
\hline & & $\begin{array}{c}\text { Incidence } \\
(\%)\end{array}$ & $\begin{array}{c}\text { Disease } \\
\text { severity } \\
(\%)\end{array}$ & $\begin{array}{c}\text { Incidence } \\
(\%)\end{array}$ & $\begin{array}{c}\text { Internal* } \\
\text { root } \\
\text { browning }\end{array}$ & $\begin{array}{c}\text { Foliar** } \\
\text { wilt } \\
\text { rating }\end{array}$ \\
\hline \multirow{7}{*}{$\begin{array}{l}\text { Solarized } \\
\text { soil }\end{array}$} & Cabbage & $4.6 \mathrm{~d}$ & $0 \mathrm{e}$ & $3.8 \mathrm{c}$ & $0.0 \mathrm{c}$ & $0.0 \mathrm{~d}$ \\
\hline & Cauliflower & $10.5 \mathrm{c}$ & $0 \mathrm{e}$ & $5.4 \mathrm{c}$ & $0.4 \mathrm{c}$ & $0.2 \mathrm{~d}$ \\
\hline & Radish & $7.5 \mathrm{~d}$ & $0 \mathrm{e}$ & $2.3 \mathrm{c}$ & $0.0 \mathrm{c}$ & $0.2 \mathrm{~d}$ \\
\hline & Canola & $11.4 \mathrm{c}$ & $2 \mathrm{e}$ & $8.6 \mathrm{bc}$ & $0.5 \mathrm{c}$ & $0.0 \mathrm{~d}$ \\
\hline & Mustard & $13.4 \mathrm{c}$ & $2 \mathrm{e}$ & $11.7 \mathrm{bc}$ & $1.2 \mathrm{c}$ & $0.2 \mathrm{~d}$ \\
\hline & Alfalfa & $12.9 \mathrm{c}$ & $4 \mathrm{e}$ & $9.5 \mathrm{bc}$ & $0.0 \mathrm{c}$ & $0.0 \mathrm{~d}$ \\
\hline & Untreated & $27.3 \mathrm{~b}$ & $44 \mathrm{~b}$ & $21.6 \mathrm{~b}$ & $6.5 \mathrm{~b}$ & $2.6 \mathrm{~b}$ \\
\hline \multirow{7}{*}{$\begin{array}{l}\text { Non- } \\
\text { solarized } \\
\text { soil }\end{array}$} & Cabbage & $13.8 \mathrm{c}$ & $10 \mathrm{de}$ & $10.6 \mathrm{bc}$ & $2.5 \mathrm{c}$ & $0.5 \mathrm{~d}$ \\
\hline & Cauliflower & $21.7 \mathrm{~b}$ & $20 \mathrm{~d}$ & $10.3 \mathrm{bc}$ & $4.2 \mathrm{bc}$ & $0.9 \mathrm{c}$ \\
\hline & Radish & $16.9 \mathrm{c}$ & $18 \mathrm{~d}$ & $8.7 \mathrm{bc}$ & $3.5 \mathrm{bc}$ & $0.3 \mathrm{~d}$ \\
\hline & Canola & $20.9 \mathrm{~b}$ & $36 \mathrm{c}$ & $15.4 \mathrm{~b}$ & $4.3 \mathrm{bc}$ & $1.0 \mathrm{c}$ \\
\hline & Mustard & $27.8 \mathrm{~b}$ & $40 \mathrm{c}$ & $21.6 \mathrm{~b}$ & $6.1 \mathrm{~b}$ & $1.8 \mathrm{~b}$ \\
\hline & Alfalfa & $29.6 \mathrm{~b}$ & $42 \mathrm{c}$ & $16.8 \mathrm{~b}$ & $5.8 \mathrm{~b}$ & $1.9 \mathrm{~b}$ \\
\hline & Untreated & $40.5 \mathrm{a}$ & $88 \mathrm{a}$ & $34.5 \mathrm{a}$ & $11.3 \mathrm{a}$ & $4.6 \mathrm{a}$ \\
\hline
\end{tabular}

* Measured as length $(\mathrm{cm})$.

** Based on scale of 0 to 5 described by Gao et al. (1995).

- Values followed by the same letter in each column are not significantly different at $\mathrm{P} \leq 0.05$ according to Duncan's multiple range tests. 

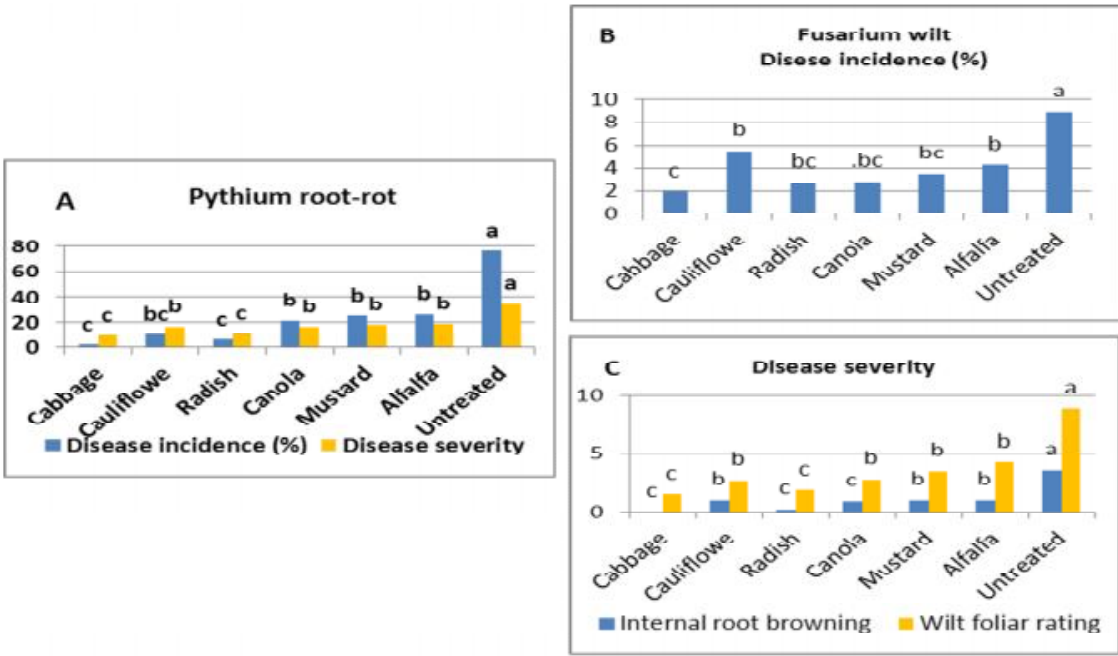

Fig. 2. Effect of crop residues on incidence of root-rot and wilt of cucumber plants grown under plastic house at Noubariya.

Results illustrated in Fig. (3) indicate that soil mulching treatment alone led to reduce the percentage of incidence and disease severity for both root-rot and wilt diseases.

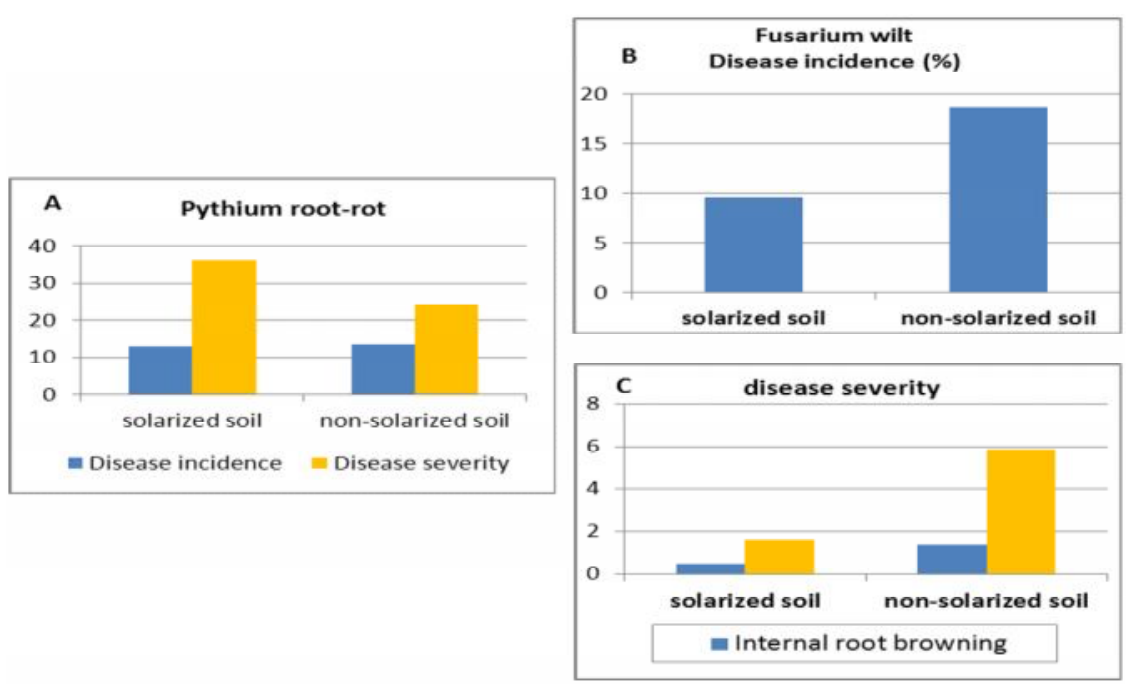

Fig. 3. Effect of solarization on incidence of root-rot and wilt of cucumber plants grown under plastic house at Noubariya. 
The combination of solarization and crop residues that, more significantly reduced both root-rot and wilt diseases than applying crop residues or solarization alone. Data in Table (3) show that there was significant effect of soil amendment with all crop residues when combined with plastic mulching on percentage of incidence and disease severity for both root-rot and wilt diseases compared with untreated control. The most reduction percentage of root-rot incidence by 88.6, $81.7 \%$ was obtained by incorporating cabbage and radish residues, respectively, in solarized soil followed by other crop residues compared with untreated control. Nevertheless, incorporation radish, cabbage and cauliflower were most significant effective to reduce incidence percentage of Fusarium wilt by 93,89 and $84.3 \%$, respectively, in solarized soil followed by other treatments compared with untreated control.

Effect of solarization and/or crop residues on microbial activity:

Population densities of selected soil microorganisms changed during solarization of soil amended with crop residues. Data in Table (4) indicate that the population density of Fusarium spp. and Pythium spp. was lower significantly in solarized soil after 60, 150 and 180days from mulching than in non- solarized soil. Nevertheless, soil amended with all crop residues led to significant gradual decrease of Fusarium spp. and Pythium spp. population densities, reaching a maximum after 150 days from treatments.

Table 4. Effect of combination of solarization and/or crop residues on pathogen's density

\begin{tabular}{|c|c|c|c|c|c|c|c|c|c|}
\hline \multirow{3}{*}{ Treatment } & \multirow{3}{*}{ Crop residue } & \multicolumn{8}{|c|}{ Days after treatment } \\
\hline & & \multicolumn{4}{|c|}{ Fusarium spp. } & \multicolumn{4}{|c|}{ Pythium spp. } \\
\hline & & 0 & 60 & 150 & 180 & 0 & 60 & 150 & 180 \\
\hline \multirow{7}{*}{$\begin{array}{l}\text { Solarized } \\
\text { soil }\end{array}$} & Cabbage & $47.4 \mathrm{a}$ & $0 \mathrm{e}$ & $0.5 \mathrm{f}$ & $0.8 \mathrm{f}$ & $51.4 \mathrm{a}$ & $0.5 \mathrm{~d}$ & $0.6 \mathrm{c}$ & $1.0 \mathrm{~d}$ \\
\hline & Cauliflower & $48.3 \mathrm{a}$ & $0 \mathrm{e}$ & $0 \mathrm{f}$ & $0.5 \mathrm{f}$ & $49.6 \mathrm{a}$ & \begin{tabular}{|l|} 
Od \\
\end{tabular} & $0 \mathrm{c}$ & Od \\
\hline & Radish & $47.4 \mathrm{a}$ & $0 \mathrm{e}$ & $0.2 \mathrm{f}$ & $0.5 \mathrm{f}$ & $50.2 \mathrm{a}$ & $0 \mathrm{~d}$ & $0 \mathrm{c}$ & $0.5 \mathrm{~d}$ \\
\hline & Cano & $47.5 \mathrm{a}$ & $0.5 \mathrm{e}$ & $0.7 \mathrm{ef}$ & $1.2 \mathrm{ef}$ & $58.9 \mathrm{a}$ & $0.5 \mathrm{~d}$ & $0.3 \mathrm{c}$ & $0.7 \mathrm{~d}$ \\
\hline & Must & $47.3 \mathrm{a}$ & $0.8 \mathrm{e}$ & $1.6 \mathrm{e}$ & $2.5 \mathrm{e}$ & $50.6 \mathrm{a}$ & $0.4 \mathrm{~d}$ & $0.3 \mathrm{c}$ & $1.4 \mathrm{~d}$ \\
\hline & Alfal & $48.5 \mathrm{a}$ & $2.8 \mathrm{~d}$ & $3.7 \mathrm{~d}$ & $6.9 \mathrm{de}$ & $51.3 \mathrm{a}$ & $2.3 \mathrm{c}$ & $2.0 \mathrm{c}$ & $4.9 \mathrm{c}$ \\
\hline & Untr & $49.6 \mathrm{a}$ & $6.5 \mathrm{~d}$ & $5.5 \mathrm{~d}$ & $11.5 \mathrm{~d}$ & $52.4 \mathrm{a}$ & $3.2 \mathrm{c}$ & $2.9 \mathrm{c}$ & $6.9 \mathrm{c}$ \\
\hline \multirow{7}{*}{$\begin{array}{l}\text { Non- } \\
\text { solarized } \\
\text { soil }\end{array}$} & $\mathrm{Cabb}$ & $47.3 \mathrm{a}$ & $31.9 \mathrm{c}$ & $25.8 \mathrm{c}$ & $34.3 \mathrm{bc}$ & $51.2 \mathrm{a}$ & $24.3 \mathrm{~b}$ & $22.4 \mathrm{~b}$ & $29.7 b$ \\
\hline & Cauliflower & $46.9 \mathrm{a}$ & $20.3 \mathrm{c}$ & $18.4 \mathrm{c}$ & $26.4 \mathrm{c}$ & $49.7 \mathrm{a}$ & $18.3 \mathrm{~b}$ & $14.2 \mathrm{~b}$ & $22.3 b$ \\
\hline & Radish & $47.3 \mathrm{a}$ & $22.6 \mathrm{c}$ & $13.4 \mathrm{c}$ & $32.9 \mathrm{c}$ & $50.6 \mathrm{a}$ & $21.6 \mathrm{~b}$ & $17.4 \mathrm{~b}$ & $28.3 b$ \\
\hline & Canc & $45.6 \mathrm{a}$ & $28.0 \mathrm{c}$ & $17.5 \mathrm{~b}$ & $34.3 \mathrm{bc}$ & $51.8 \mathrm{a}$ & $26.4 \mathrm{~b}$ & $20.5 b$ & $30.2 b$ \\
\hline & Mustard & $46.6 \mathrm{a}$ & $31.8 \mathrm{~b}$ & $23.5 \mathrm{~b}$ & $35.1 \mathrm{~b}$ & $52.4 \mathrm{a}$ & $31.7 \mathrm{~b}$ & $26.4 b$ & $35.3 b$ \\
\hline & Alfalfa & $48.5 \mathrm{a}$ & $35.5 \mathrm{~b}$ & $26.9 \mathrm{~b}$ & $37.4 \mathrm{~b}$ & $49.8 \mathrm{a}$ & $33.1 \mathrm{~b}$ & $30.3 b$ & $34.7 \mathrm{~b}$ \\
\hline & Untreated & $47.6 \mathrm{a}$ & $50.1 \mathrm{a}$ & $55.3 \mathrm{a}$ & $57.2 \mathrm{a}$ & $54.3 a$ & $57.5 \mathrm{a}$ & $68.9 \mathrm{a}$ & 72.6 \\
\hline
\end{tabular}

Values followed by the same letter in each column are not significantly different at $\mathrm{P} \leq 0.05$ according to Duncan's multiple range tests. 
While, there was more significant effect of incorporated crop residues when combined with soil mulching on inoculums densities of these pathogens. Incorporation of cabbage, radish and cauliflower followed by plastic mulching decreased Fusarium spp. and Pythium spp. population compared with either crop residues or solarization alone.

The present data in (Table 5) show that gradual increase was recorded in population of bacteria as a result of soil amendment with cabbage, radish, cauliflower, canola, mustard and alfalfa compared with untreated control in nonsolarized soil. This increasing reached its maximum after 150 days from start of treatments ( 2 months after transplanting cucumber), then it was deadline slightly. The pronounced increase in bacterial population was recorded with treatments of alfalfa, canola and mustard in non-solarized soil. Bacterial total count decreased in solarized soil, the observation for population of some benefit bacteria such as, spore forming bacteria (Bacillus spp.) and fluorescent Pseudomonas were stable in soil plots where mulching. Similar trend of results were recorded with populations of actinomycetes significantly there was no deference of numbers of actinomycetes population in ether non-solarized soil or solarized soil. Meanwhile, population densities of thermotolerant fungi, such as Penicillium spp., Aspergillus spp. and Trichoderma spp., increased significantly in solarized soil.

Table 5. Effect of combination of solarization or/ and crop residues on total count of microorganisms

\begin{tabular}{|c|c|c|c|c|c|c|c|c|c|c|c|c|c|}
\hline \multirow{3}{*}{ Treatment } & \multirow{3}{*}{$\begin{array}{l}\text { Crop } \\
\text { residue }\end{array}$} & \multicolumn{12}{|c|}{ Days after treatment } \\
\hline & & \multicolumn{4}{|c|}{ Bacteria } & \multicolumn{4}{|c|}{ Fungi } & \multicolumn{4}{|c|}{ Actinomycetes } \\
\hline & & 0 & 60 & 150 & 180 & 0 & 60 & 150 & 180 & 0 & 60 & 150 & 180 \\
\hline \multirow{7}{*}{$\begin{array}{l}\text { Solarized } \\
\text { soil }\end{array}$} & Cabbage & $8 \mathrm{a}$ & $28 \mathrm{c}$ & $32 \mathrm{e}$ & $21 \mathrm{e}$ & $3 a$ & $4 \mathrm{c}$ & $13 \mathrm{c}$ & $5 c$ & $3 a$ & $6 b$ & $10 \mathrm{~b}$ & $6 \mathrm{~b}$ \\
\hline & Cauliflower & $9 \mathrm{a}$ & $25 \mathrm{c}$ & $41 d$ & $32 \mathrm{e}$ & $4 a$ & $5 c$ & $11 \mathrm{c}$ & $7 \mathrm{c}$ & $3 a$ & $4 \mathrm{~b}$ & $7 b$ & $5 b$ \\
\hline & Radish & $9 a$ & $28 \mathrm{c}$ & $45 \mathrm{~d}$ & $24 \mathrm{e}$ & $4 \mathrm{a}$ & $6 c$ & $9 \mathrm{c}$ & $8 \mathrm{c}$ & $4 \mathrm{a}$ & $4 \mathrm{~b}$ & $6 b$ & $5 b$ \\
\hline & Canola & $9 a$ & $39 b$ & $68 \mathrm{c}$ & $30 \mathrm{e}$ & $3 a$ & $9 b$ & $21 b$ & $11 b$ & $3 a$ & $4 \mathrm{~b}$ & $13 \mathrm{a}$ & $5 b$ \\
\hline & Mustard & $8 \mathrm{a}$ & $38 b$ & $51 d$ & $38 d$ & $4 \mathrm{a}$ & $7 \mathrm{c}$ & $18 \mathrm{~b}$ & $10 \mathrm{~b}$ & $3 a$ & $7 \mathrm{~b}$ & $9 b$ & $5 b$ \\
\hline & Alfalfa & $9 a$ & $49 b$ & $114 \mathrm{a}$ & $74 b$ & $4 a$ & $15 b$ & $29 \mathrm{ab}$ & $12 b$ & $4 a$ & $12 \mathrm{a}$ & $23 a$ & $10 \mathrm{a}$ \\
\hline & Untreated & $8 \mathrm{a}$ & $4 d$ & $5 \mathrm{e}$ & $3 f$ & $4 a$ & $4 c$ & $4 d$ & $3 c$ & $3 a$ & $5 b$ & $4 \mathrm{c}$ & $4 \mathrm{~b}$ \\
\hline \multirow{7}{*}{$\begin{array}{l}\text { Non- } \\
\text { solarized } \\
\text { soil }\end{array}$} & Cabbage & $9 a$ & $43 b$ & $69 c$ & $41 d$ & $4 a$ & $7 \mathrm{c}$ & $18 \mathrm{~b}$ & $13 b$ & $3 a$ & $4 \mathrm{~b}$ & $9 b$ & $7 b$ \\
\hline & Cauliflower & $10 \mathrm{a}$ & $40 b$ & $71 \mathrm{c}$ & $49 \mathrm{c}$ & $5 a$ & $10 \mathrm{~b}$ & $23 b$ & $15 b$ & $3 a$ & $7 b$ & $11 \mathrm{~b}$ & $6 \mathrm{~b}$ \\
\hline & Radish & $9 \mathrm{a}$ & $39 b$ & $75 c$ & $42 d$ & $4 a$ & $9 b$ & $19 b$ & $12 b$ & $4 a$ & $5 b$ & $8 b$ & $5 b$ \\
\hline & Canola & $9 a$ & $48 \mathrm{~b}$ & $80 \mathrm{~b}$ & $55 \mathrm{c}$ & $4 a$ & $15 b$ & $30 a$ & $22 a$ & $2 a$ & $5 b$ & $15 b$ & $7 b$ \\
\hline & Mustard & $9 a$ & $54 a$ & $77 b$ & $50 \mathrm{c}$ & $5 a$ & $11 \mathrm{~b}$ & $26 a$ & $19 \mathrm{a}$ & $3 a$ & $8 b$ & $11 \mathrm{~b}$ & $6 b$ \\
\hline & \begin{tabular}{|l|} 
Alfalfa \\
\end{tabular} & $10 \mathrm{a}$ & $61 \mathrm{a}$ & $140 \mathrm{a}$ & $90 \mathrm{a}$ & $4 a$ & $22 a$ & $35 \mathrm{a}$ & $20 a$ & $4 a$ & $15 \mathrm{a}$ & $24 a$ & $12 \mathrm{a}$ \\
\hline & Untreated & $9 \mathrm{a}$ & $10 \mathrm{~d}$ & $9 \mathrm{e}$ & $8 \mathrm{f}$ & $3 a$ & $5 c$ & $5 d$ & $4 \mathrm{c}$ & $3 a$ & $4 \mathrm{~b}$ & $4 \mathrm{c}$ & $5 b$ \\
\hline
\end{tabular}

Values followed by the same letter in each column are not significantly different at $\mathrm{P} \leq 0.05$ according to Duncan's multiple range tests. 
Effect of solarization and/or crop residues on cucumber growth parameters

Data presented in Table (6) indicate that either all crop residues or solarization increased growth parameters, i.e. plant height, fresh and dry weights as well as fruit yield of cucumber plants compared with untreated control. Alfalfa was the most significant effect to increase plant height, fresh and dry weights followed by cabbage, canola and mustard treatments. This increment observed in solarized soil better than non solarized soil. Meanwhile, Radish and Cauliflower treatments gave low significant effect increasing growth parameters of cucumber plants. Also, data in Table (6) show that all croup residue treatments increased fruit yield of cucumber in solarized soil than non-solarized soil compared with untreated control. The most significant effective treatment was alfalfa followed by cabbage; canola and mustered treatments for increasing fruit yield of cucumber plants were grown in solarized soil compared with untreated control.

Table 6. Effect of combination solarization and crop residues treatments on Cucumber growth parameters

\begin{tabular}{|l|l|c|c|c|c|}
\hline \multirow{2}{*}{ Treatment } & \multirow{2}{*}{ Crop residue } & \multicolumn{3}{|c|}{ Growth character } & Fruit yield* \\
\cline { 3 - 6 } & & Plant height & Fresh weight & Dry weight & (ton/field) \\
\hline \multirow{5}{*}{ Solarized soil } & Cabbage & $74.3 \mathrm{~b}$ & $295 \mathrm{~b}$ & $115 \mathrm{a}$ & $2.6 \mathrm{ab}$ \\
\cline { 2 - 6 } & Cauliflower & $65.8 \mathrm{bc}$ & $268 \mathrm{~b}$ & $90 \mathrm{~b}$ & $1.8 \mathrm{~b}$ \\
\cline { 2 - 6 } & Radish & $63.6 \mathrm{bc}$ & $260 \mathrm{~b}$ & $85 \mathrm{~b}$ & $1.7 \mathrm{~b}$ \\
\cline { 2 - 6 } & Canola & $72.7 \mathrm{~b}$ & $322 \mathrm{a}$ & $113 \mathrm{a}$ & $2.5 \mathrm{ab}$ \\
\cline { 2 - 6 } & Mustard & $70.5 \mathrm{~b}$ & $321 \mathrm{a}$ & $111 \mathrm{a}$ & $2.7 \mathrm{ab}$ \\
\cline { 2 - 6 } & Alfalfa & $85.7 \mathrm{a}$ & $340 \mathrm{a}$ & $120 \mathrm{a}$ & $3.5 \mathrm{a}$ \\
\cline { 2 - 6 } & Untreated & $58.3 \mathrm{c}$ & $220 \mathrm{c}$ & $87.2 \mathrm{~b}$ & $0.9 \mathrm{c}$ \\
\hline \multirow{5}{*}{$\begin{array}{l}\text { Non-solarized } \\
\text { soil }\end{array}$} & Cabbage & $67.0 \mathrm{bc}$ & $231 \mathrm{c}$ & $92 \mathrm{~b}$ & $2.2 \mathrm{~b}$ \\
\cline { 2 - 6 } & Cauliflower & $56.4 \mathrm{c}$ & $200 \mathrm{c}$ & $71 \mathrm{c}$ & $1.7 \mathrm{~b}$ \\
\cline { 2 - 6 } & Radish & $55.6 \mathrm{c}$ & $194 \mathrm{c}$ & $70 \mathrm{c}$ & $1.6 \mathrm{~b}$ \\
\cline { 2 - 6 } & Canola & $63.9 \mathrm{bc}$ & $289 \mathrm{~b}$ & $92 \mathrm{~b}$ & $2.1 \mathrm{~b}$ \\
\cline { 2 - 6 } & Mustard & $64.5 \mathrm{bc}$ & $292 \mathrm{~b}$ & $95 \mathrm{~b}$ & $1.9 \mathrm{~b}$ \\
\cline { 2 - 6 } & Alfalfa & $73.9 \mathrm{~b}$ & $326 \mathrm{a}$ & $112 \mathrm{a}$ & $3.0 \mathrm{a}$ \\
\cline { 2 - 6 } & Untreated & $40.5 \mathrm{~d}$ & $141 \mathrm{~d}$ & $49.4 \mathrm{~d}$ & $0.4 \mathrm{c}$ \\
\hline
\end{tabular}

*Cucumber fruit yield was determined at the end of growing season (three months).

- Values followed by the same letter in each column are not significantly different at $\mathrm{P} \leq 0.05$ according to Duncan's multiple range tests.

\section{D i s c u s s i o n}

Soil solarization is a natural hydrothermal process of disinfesting soil of soil borne pathogens in subtropical and southern desert regions, which is accomplished through passive solar heating. Solarization occurs through a combination of physical, chemical and biological mechanisms, and is compatible with many other disinfestation methods to provide integrated pest management (Stapleton, 2000; Klein et al., 2011; Katan and Gamliel, 2012 and Parvatha Reddy, 2013). 
Efficacy of soil solarization for control the soil borne pathogens is a function of time and temperature relationships. Results of the present study indicated that, soil temperatures under mulching with polyethylene sheet during summer season 2009 was increased significantly; to reach $51^{\circ} \mathrm{C}$ at $5 \mathrm{~cm}$ depth and $44^{\circ} \mathrm{C}$ at $20 \mathrm{~cm}$ depth. Soil solarization is based on the exploitation the solar energy for heating wet soil mulched with transparent Polyethylene sheets to $40-55^{\circ} \mathrm{C}$ in the upper soil layer. The highest temperatures during solarization were archived on near the soil surface. Soil temperature decreases with increasing depth. Generally, temperatures commonly reach to $35-60^{\circ} \mathrm{C}$ during soil solarization, depending on soil type, season, location, soil depth and other factors (Stapleton, 2000).

In this study, Pythium root-rot and Fusarium wilt were decreased by more than $45 \%$ in solarized soil compared with non-solarized soil. Although, numbers of propagules of Pythium sp. and Fusarium spp. were reduced in solarized soil more than non-solarized soil. It is based on fact that most plant pathogens are mesophylic. Mesophylic organisms have an upper temperature threshold of about $37^{\circ} \mathrm{C}$; accumulated heat effects at this or higher temperatures over time are lethal. The reduction of propagule numbers due to direct thermal inactivation of soil borne pathogens which is the most obvious and important mechanisms of the solarization process. Fungal propagules exposed to sub lethal heat are weakened, which leads to reduced viability and susceptibility to competition or antagonistic activity of the native soil microflora (Stapleton, 2000). Soil solarization also induces changes in soil volatile compounds which will also be toxic to organisms already weakened by high temperature.

Solarization has been proved to be effective in controlling populations of many important soil borne fungal pathogens such as Verticillium dahliae, the causal agent of vascular diseases of many plants (Pinkerton et al., 2000), certain Fusarium spp. that cause root-rot and wilt in several crops (Ramirez-Villapudua and Munnecke, 1987; 1988 and Klein et al., 2011); Pythium aphanidermatum (Deadman et al., 2006); Phytophthora cinnamomi (Coelho et al., 1999) and Rhizoctonia solani (Pullman et al.,1981). Moreover, Solarization causes important biological changes in treated soils. Results indicated that in solarized soil, the total count of fungi and bacteria were decreased. Yet, was stable of actinomycetes, counts and some species of bacterial and fungal counts. The destruction of many mesophilic microorganisms during solarization creates a partial biological vacuum in which substrate and nutrients in soil are made available for re-colonization following treatment. Although, most mesophilic organisms in soil have thermal damage thresholds beginning around $39-40^{\circ} \mathrm{C}$, some thermophilic and thermotolerant organisms can survive temperatures archived in most types of solarization treatment (Stapleton and DeVay, 1995). Solarization initially may reduce populations of beneficial microorganisms, but populations of beneficial, growth-promoting and pathogenantagonistic bacteria and fungi quickly re-colonize solarized soil (Gamliel and Katan, 1999). Thus, soil solarization adds a biological control component (Gamliel and Katan, 2009). Plant-pathogenic fungi weakened by high soil temperatures are more susceptible to these antagonists (Katan, 1987 and Stapleton and DeVay, 1995). Saprophytic microorganisms, including several antagonists, are usually more tolerant to heat than plant pathogens (Stapleton, 2000).

Egypt. J. Phytopathol., Vol. 41, No.1 (2013) 
The use of organic amendments (biofumigation) such incorporated cover crop residues was effective to suppress soil borne pathogens (Karavina and Mondumbu, 2012 and Hansen and Keinath, 2013). Combining crop residues with soil solarization increases efficacy of soil borne pathogen management control (Pokharel and Hammon, 2010). Results in this study illustrated that, the crop residue treatments had the biocidal effect to reduce the incidence and disease severity of both Pythium root-rot and Fusarium wilt diseases of cucumber. However, combination crop residue treatments and mulching soil with polyethylene sheets had more reduction effect for these diseases. Cabbage, radish and Cauliflower residues were the most effective treatments for reducing these diseases in solarized soil compared with non-solarized soil. Organic matter addition increased the rate of decomposition of these materials in the soil and thereby the rate of heat generation during decomposition; it also increases the heat-carrying capacity of the soil. It may further elevate the soil temperature by an additional 1-3 ${ }^{\circ} \mathrm{C}$ (Gamliel and Stapleton, 1993a \& 1993b; Gamliel, et al., 2000 and Lira-Saldivar et al., 2004).

The benefit of combining organic amendments with solarization is that pathogen populations can be reduced at soil temperature lower than those required without organic matter additions. Improving soil solarization efficacy by organic amendments leads to the generation of toxic volatile compounds that accumulate under the plastic mulch and consequently enhance the vulnerability of soil organisms to soil solarization (Gamliel et al., 2000).

The nature of these volatiles may vary according to the origin of the organic matter (Wheatley et al., 1996), especially when a high soil temperature is employed (Gamliel and Stapleton, 1993a \& 1993b and Gamliel et al., 2000). Different plant residues incorporated into solarized soil may generate measurable amounts of volatiles such as ammonia, methanethiol, dimethyl sulfide, under the polyethylene sheet to above a threshold level that is toxic to soil flora and fauna. The elevated soil temperature also increases the sensitivity of soil borne pathogens to the toxic effect of the captured volatiles (Gamliel et al., 2000). In addition, soil treatment with organic amendments followed by soil solarization may be effective against natural soil populations of the damping off fungi Pythium ultimum and Verticillium dahlia (Pullman et al., 1981 and Stapleton, 2000).

Solarization appears to be an effective practice able to control soil borne pathogens, even though it may cause temporary stress on some beneficial soil microbial biomass. Organic soil amendments can protect soil microbial biomass and enzymatic activities from the detrimental effect of heating. Brassica spp. are known to have volatile compounds such as glucosinolates in their leaves, stems and roots that can be hydrolyzed to produce isothiocyanates that have fungicidal (Angus et al., 1994; Karavina and Mondumbu, 2012; Hansen and Keinath, 2013 and Omirou et al., 2013). In this study, in solarized soil crop residues- amended was more effective in reducing the populations of Pythium sp. and Fusarium spp., as has also been shown in other studies (Pullman et al., 1981; Deadman et al., 2006 and Klein et al., 2011). Numbers of propagules were reduced when the propagules were exposed to volatile compounds from crop residues in solarized soil. 
Results in this study illustrated that, crucifer residues have more reduction effective for populations of Fusarium spp. and Pythium sp. when combined with solarized soil. Similar results were obtained, soil heating and amendment with cabbage residues reduced propagule numbers of Pythium ultimum, Sclerotium rolfsii and Fusarium oxysporum due to the level of isothiocyanates and aldehydes which generated (Gamliel and Stapleton, 1993b).

However, results showed that all crop residue treatments increased total count of microorganisms as: bacteria, fungi and actinomycetes in solarized or non-solarized soil. Additions of organic matter often stimulate activity of microorganisms that are antagonistic pathogens (Klein et al., 2013). Indirect effects on the pathogens associated with changes in the populations of antagonistic organisms, as well as effects of compounds released from the tissues (Mazzola, 2004; Matthiessen and Kirkegaard, 2006 and Liu et al., 2007).

Results in this study demonstrated that, cucumber growth characters significantly were increased in solarized soil better than non-solarized soil. Chemical mechanisms occur during solarization, increase in concentration of soluble mineral materials commonly observed following treatment (Stapleton, 2000). Stapleton and DeVay (1995) showed that in soil types ranging from heavy sand to silty clay, NH4-N and NO3-N concentration in the top $15 \mathrm{~cm}$ soil depth were increased. Concentrations of other soluble mineral nutrients, including calcium, magnesium, phosphorus, potassium and others also sometimes increased.

Increasing in available mineral nutrients in soil can play a major role in the effect of solarization, leading to increase plant health and growth, and reduce fertilization requirement (Ibarra-Jimenez et al., 2012). Increases in some of the mineral nutrient concentrations can be attributed to composition of organic component of soil during treatment. Improved mineral nutrition is also often associated with soil fumigation (Chen et al., 1991).

\section{Con c l u s i o n}

As a final conclusion of this study, it is highly recommended to use soil solarization in combination with crop residues as "biofumigation" to improve control of cucumber soil borne pathogens. This will result in better environmental and conditions and habitat conservation. In addition better results will obtained from biofumigation compared to the ordinary fumigation.

\section{Ref e r e n e s}

Abd El-Hafez, A.M. 1966. Some studies on acid producing microorganisms in soil and rhizosphere with special reference to phosphate dissolvers. Ph.D. Thesis, Fac. Agric., Ain Shams Univ., Egypt.

Angus, J.F.; Gardner, P.A.; Kirkegaard, J.A. and Desmarchelier, J.M. 1994. Biofumigation isothiocyanates released from Brassica roots inhibit growth of the take-all fungus. Plant and Soil, 162: 107-112. 
Anita, B. 2012. Crucifer vegetable leaf wastes as biofumigants for the management of root knot nematode (Meloidogyne hapla Chit Wood) in celery (Apium graveolens L.). J. Biopest., 5: 111-114.

Barnett, H.L. and Hunter, B.B. 1998. Illustrated Genera of Imperfect Fungi. $4^{\text {th }}$ Ed. APS Press, St. Paul, Minn., USA, 241pp.

Berbegal, M.; García-Jiménez, J. and Armengol, J. 2008. Effect of cauliflower residue amendments and soil solarization on Verticillium wilt control in Artichoke. Plant Dis., 92: 595-600.

Chellemi, D.O.; Olson, S.M.; Mitchell, D.J.; Secker, I. and McSorley, R. 1997. Adaptation of soil solarization to the integrated management of soil borne pests of tomato under humid conditions. Phytopathology, 87: 250-258.

Chen, Y.; Gamliel, A.; Stapleton, J.J. and Aviad, T. 1991. Chemical, physical, and microbial changes related to plant growth in disinfested soils. Pages: 103-129. In: Soil Solarization. Katan, J. and DeVay, J.E. (Eds.), CRC Press, Boca Raton.

Coelho, L., Chellemi, D.O. and Mitchell, D.J. 1999. Efficacy of solarization and cabbage amendment for the control of Phytophthora spp. in North Florida. Plant Dis., 83: 293-299.

Deadman, M.; Al-Hasani, H. and Al-Sa'di, A. 2006. Solarization and biofumigation reduce Pythium aphanidermtum induced damping-off and enhance vegetative growth of greenhouse cucumber in Oman. J. Plant Pathol., 88: 335-337.

Duncan, D.B. 1955. Multiple range and multiple F tests. Biometrics, 11: 1-42.

Flowers, R.A. and Hendrix, J.W. 1969. Gallic acid in a procedure for isolation of Phytophthora parasitica var. nicotianane and Pythium spp. from soil. Phytopathology, 59: 725-731.

Gamliel, A. and Stapleton, J.J. 1993a. Effect of soil amendment with chicken compost or ammonium phosphate and solarization on pathogen control, rhizosphere microorganisms and lettuce growth. Plant Dis., 77: 886-891.

Gamliel, A. and Stapleton, J.J. 1993b. Characterization of antifungal volatile compounds evolved from solarized soil amended with cabbage residues. Phytopathology, 83: 899-905

Gamliel, A. and Katan, J. 1999. Involvement of fluorescent Pseudomonads and other microorganisms increased growth response of plants in solarized soil. Phytopathology, 81: 494-502.

Gamliel, A. and Katan, J. 2009. Control of plant disease through soil solarization. Pages: 196-220. In: Disease Control in Crops. D. Walters (ed.). Blackwell Publishing LTd, Oxford.

Gamliel, A.; Austeraweil, M. and Kritzman, M. 2000. Non-chemical approach to soil borne pest management-organic amendments. Crop Protection, 19: 847-853. 
Gao, H.; Beckman, C.H. and Mueller, W.C. 1995. The rate of vascular colonization as a measure of the genotypic interaction between various cultivars of tomato and various formae or races of Fusarium oxysporum. Physiol. Mol. Plant Pathol., 46: 29-43.

Gilman, J.C. 1957. A Manual of Soil Fungi. Iowa State Univ. Press, Ames. Iowa, U.S.A. $418 p p$.

Hansen, Z.R. and Keinath, A.P. 2013. Increased pepper yields following incorporation biofumigation cover crops and the effects on soil borne pathogen populations and pepper diseases. Appl. Soil Ecol., 63: 67-77.

Ibarra-Jimenez, L.; Lira Saldivar, H.; Cardenas- Flores, A. and Valdez-Aguilar, L. 2012. Soil solarization enhances growth and yield in dry beans. Acta Agric. Scandinavia, 6: 541-546.

Karavina, C. and Mondumbu, R. 2012. Biofumigation for crop protection: Potential for adoption in Zimbabwe. J. Animal \& Plant Sci., 14: 1996-2005.

Katan, J. 1987. Soil solarization. Pages: 7-105. In: Innovative Approaches to Plant Disease Control. Chet, I. (ed.). John Wiley and Sons Inc., NY, USA. 372pp.

Katan, J. and Gamliel, A. 2012. Mechanisms of pathogen and disease control and plant growth important involved in soil solarization In: Soil Solarization: Theory and Practice. A. Gameliel and J. Katan (eds.). APS. Press, St. Paul, MN, USA. 280pp.

Klein, E.; Katan, J.; Austerweil, M. and Gamliel, A. 2007. Controlled laboratory system to study soil solarization and organic amendment effects on plant pathogens. Phytopathology, 97: 1476-1483.

Klein, E; Katan, J. and Gamliel. A. 2011. Soil suppressiveness to Fusarium disease following organic amendments and solarization. Plant Dis., 95: 1116-1123.

Klein, E.; Ofek, M.; Katan, J.; Minz, D. and Gamliel, A. 2013. Soil suppressiveness to Fusarium disease: shifts in root microbiome associated with reduction of pathogen root colonization. Phytopathology, 103 (1): 23-33.

Komada, H. 1975. Development of selective medium for quantitative isolation of Fusarium oxysporum from natural soils. Rev. Plant Protec. Res., 8: 114-124.

Lira-Saldivar, R.H.; Salas, M.A.; Cruz, J.; Coronado, F.D.; Guerrero, E. and Gallegos, G. 2004. Solarization and goat manure on weeds management and melon yield. Phyton, 53: 205-211.

Liu, B.; Gumpertz, M.L. and Ristanio, J.B. 2007. Long- term effects of organic and synthetic soil fertility amendments on soil microbial communities and the development of southern blight. Soil Biol. Biochem., 39: 2302-2316.

Marois, J.J.; Mitchell, D.J. and Sonoda, R.M. 1981. Biological control of Fusarium crown rot of tomato under field conditions. Phytopathology, 71: 1257-1260. 
Matthiessen, J.N. and Kirkegaard, J.A. 2006. Biofumigation and enhanced biodegradation: opportunity and challenge in soil borne pest and disease management. Critical Rev. Plant Sci., 25: 235-265.

Mazzola, M. 2004. Assessment and management of soil microbial community structure for disease suppression. Annu. Rev. Phytopathol., 42: 35-59.

Omirou, M.; Karpouzas, D.; Papadopoulou, K. and Ehaliotis, C. 2013. Dissipation of pure and broccoli- release glucosinolates in soil under high and low moisture content. Europ. J. Soil Biol., 60: 62-70.

Parvatha Reddy, P. 2013. Soil solarization. Pages: 159-183. In: Recent Advances in Crop Protection. P. Parvatha Reddy (ed.). Publishing Springer India.

Pinkerton., J.N. 2000. Effect of soil solarization and cover crops on populations of selected soil borne plant pathogens in Western Oregon. Plant Dis., 84: 952-959.

Pokharel, R. and Hammon, R. 2010. Increase efficacy of biofumigation by soil solarization and integrating with Brassica meal cake and poultry manure to manage soil borne problem in onion. Report Submitted to EPA, PESP program. $23 \mathrm{pp}$.

Pullman, G.S.; DeVay, J.E.; Garber, R.H. and Weinhold, A.R. 1981. Soil solarization: Effect on Verticillium wilt of cotton and soil borne populations of Verticillium dahlia, Pythium spp., Rhizoctonia solani and Thielaviopsis basicola. Phytopathology, 7I: 954-959.

Ramirez-Villapudua, J. and Munnecke, D.M. 1987. Control of cabbage yellows (Fusarium oxysporum f. sp. conglutinans) by solar heating of soils amended with dry cabbage residues. Plant Dis., 71: 217-221.

Ramirez-Villapudua, J. and Munnecke, D.M. 1988. Effect of solar heating and soil amendments of cruciferous residues on Fusarium oxysporum f.sp. conglutinans and other organisms. Phytopathology, 78:289-295.

Stapleton, J.J. 1997. Solarization: An implementable alternative for soil disinfestation. Pages: 1-6. In: Biological and Cultural Tests for Control of Plant Diseases, Vol. 12. Canaday, C. (ed.). APS Press, St. Paul, MN, USA.

Stapleton, J.J. 2000. Soil solarization in various agricultural production systems. Crop Protection, 19: 837-841.

Stapleton, J.J.; DeVay, J.E. 1995. Soil solarization: A natural mechanism of integrated pest management. Pages: 309-322. In: Novel Approaches to Integrated Pest Management. Reuveni, R. (ed.). Lewis Publishers, Boca Raton.

Stapleton, J.J.; DeVay, J.E. and Lear, B. 1991. Simulated and effect of ammoniabased fertilizers and soil solarization on pathogen survival, soil fertility and crop growth. Pages: 331-342. In: Soil Solarization. DeVay, J.E.; Stapleton, J.J.; Elmore, C.L. (eds.). CRC Press, Boca Raton.

Szczech, M.M. 1999. Suppressiveness of vermicompost against Fusarium wilt of tomato. J. Phytopathol., 147: 155-161. 
Waksman, S.M. 1957. Soil microbiology. John Willy and Sons, New York and London.

Wheatley, R.H.; Millar, S.E. and Griffiths, D.W. 1996. The production of volatile organic compounds during nitrogen transformations in soils. Plant and Soil, 181: 163-167.

(Received 17/03/2013; in revised form 22/04/2013)

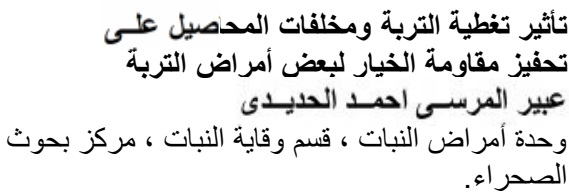

تتعرض محاصيل الخضر المنزرعة تحت نظم الزراعات المحمية خاصة محصول

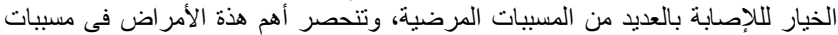

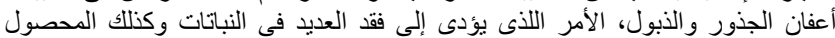

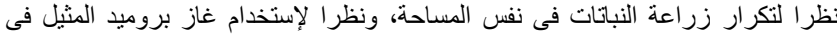

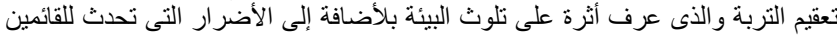

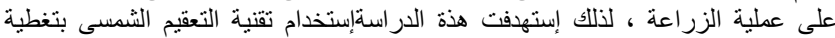

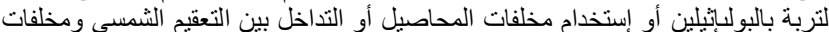
المحصول لخفض أمر اض الذبول و عفن الجذور للخيار.

يعتبر تطبيق مخلفات المحصول كاضافات تربة منبو عا بتغطية التربة بالبولبإثبلين

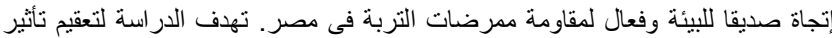

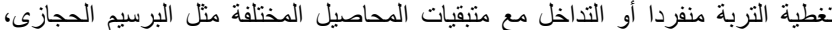

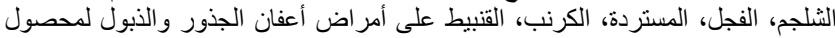

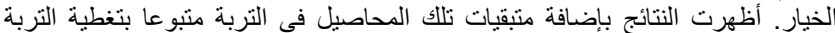

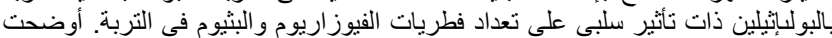

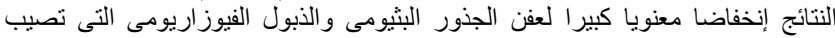

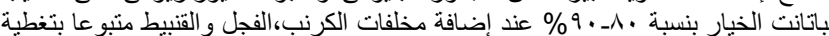

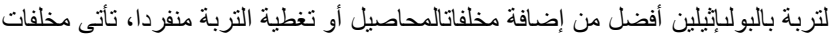

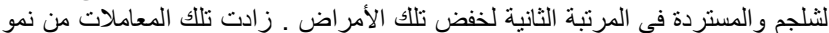
النبات و المحصول، بلإضافة إلى حدوثة زيادة الكيائينات الدقيقة فى التربة. 